\title{
Nuevas perspectivas para la historia del pueblo pijao, siglos XVI y XVII*
}

New Perspectives for the History of the Pijao People, I6th and I7th Centuries

DOI: https://doi.org/I0.22380/20274688.II25

Recibido: 26 de enero del 2020

Aprobado: 12 de marzo del 2020
JUAN JOSÉ VELÁSQUEZ ARANGO**

jjvelasquez2@hotmail.com

\section{R E S U M E N}

A pesar de las muchas menciones que la historiografía tradicional ha hecho del pueblo pijao y la guerra que protagonizó entre los siglos XVI y XVII, es muy poco lo que se conoce acerca de su sociedad, sus costumbres y su modo de subsistencia. Ante tal panorama, este artículo presenta al lector nuevas perspectivas al respecto, estudiando factores como la localización geográfica, la forma de habitación, la demografía en diferentes periodos y la organización social, entre otros, que permitan llegar a una mejor comprensión del devenir histórico de este pueblo indígena y los fenómenos en los que estuvo involucrado. Para esta investigación se utilizaron diferentes fuentes documentales de archivos nacionales e internacionales, así como investigaciones históricas y arqueológicas clásicas y recientes que, desde una perspectiva multidisciplinar, pueden ofrecer nuevos puntos de vista y temas de estudio relativos a los grupos indígenas de la actual Colombia durante los siglos XVI y XVII.

Palabras clave: pijaos, siglo XVI, siglo XVII, sociedad, costumbres

* Este artículo nace como parte de la tesis presentada en el 2018 por el autor para optar al título de magíster en Historia en la Universidad Nacional de Colombia, sede Medellín, titulada "La guerra contra los indígenas pijaos: financiamiento, organización militar y vida cotidiana, 1550-1615”.

** Historiador y magíster en Historia de la Universidad Nacional de Colombia, sede Medellín. Sus intereses giran en torno a la historia militar y social del Nuevo Reino de Granada y la gobernación de Popayán durante los siglos XVI y XVII. 


\section{$\begin{array}{llllllll}\mathbf{A} & \mathbf{B} & \mathbf{S} & \mathbf{T} & \mathbf{R} & \mathbf{A} & \mathbf{C} & \mathbf{T}\end{array}$}

Despite the many mentions that traditional historiography has made about the Pijao people about the war that they carried out between the 16th and 17th centuries, very little is known about their society, customs and way of subsistence. Considering this, the article presents new perspectives in this regard, studying factors such as geographic location, form of habitation, demography in different periods, and social organization, among others, that allow to get a better under- standing of the historical evolution of this indigenous group and the phenomena in which they were involved. For this research, different documentary sources from national and international archives were used, as well as classical and recent historical and archaeological research, which, through a multidisciplinary perspective, can offer new points of view and study topics related to indigenous groups in the current Colombia during the sixteenth and seventeenth centuries.

Keywords: pijaos, 16 th century, 17 th century, society, customs

\section{Introducción}

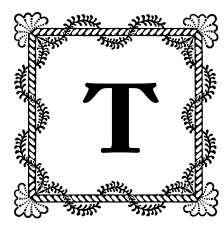

anto en la historiografía tradicional como en la cultura popular, los pijaos han sido uno de los pueblos indígenas de la actual Colombia que más atención han recibido, ya sea para crear sentimientos de identificación regional, como en el caso del departamento del Tolima, o bien para ensalzar las labores “civilizadoras” del régimen español (Restrepo 730-737). En el ámbito académico han sido estudiados en especial en torno al conflicto que desde mediados del siglo XVI hasta la segunda década del XVII mantuvieron con las tropas enviadas por las autoridades de la Audiencia de Santa Fe y la gobernación de Popayán. Sin embargo, han sido pocos los trabajos dedicados enteramente al estudio y la comprensión de su sociedad que contemplen factores como su organización social, sus costumbres, su demografía y sus formas de subsistencia, de manera aislada de la relación con la guerra mencionada, la cual si bien no puede obviarse, no cubre todo el espectro de lo que fue este pueblo. Tal vez los únicos intentos han sido los trabajos de Julio Cesar Cubillos y Manuel Lucena Salmoral, publicados en 1946 y 1965 , respectivamente, los cuales como resulta evidente por el tiempo transcurrido requieren una crítica y actualización.

De acuerdo con las precedentes consideraciones, este artículo procura aportar información y nuevas perspectivas para el estudio de este grupo indígena 
como una sociedad compleja y con dinámicas propias muchas veces opacadas por la fijación de los historiadores en su comportamiento guerrero. Para la investigación, además de una amplia bibliografía que contempla estudios y perspectivas de autores nacionales y extranjeros, se han utilizado las crónicas de fray Pedro Simón y fray Pedro de Aguado, así como diferentes fuentes de archivo provenientes del Archivo General de Indias, el Archivo General de la Nación y el Archivo Central del Cauca.

\section{Los pijaos: nombre y ubicación}

El término pijao, una aparente deformación española del nombre nativo pinao, hace referencia a un conglomerado de tribus que algunos investigadores han identificado como pertenecientes a un subgrupo de la gran familia lingüística karib que habitaron en la cordillera Central' ${ }^{1}$, en una región que comprende, aproximadamente, los siguientes límites (Cubillos, "Apuntes" 47-48; Lucena 96): al norte, desde Ibagué y el río Coello hasta la ciudad de Cartago (hoy Pereira). En el sur, desde la desembocadura del río Páez en el Magdalena, hasta la falda occidental de la cordillera Central, a la altura de Caloto. Por el oeste, la banda occidental de la cordillera Central desde Cartago hasta Caloto. Por el oriente, el río Magdalena desde la desembocadura del Páez hasta la del Coello (AGI, P, I96, R. 27, ff. 98 v.-989 r.; Arango 388; Cubillos, "Apuntes" 50-52; Friede I69; Lucena 97-I00; Simón 328-329) (véase figura I).

Según investigaciones arqueológicas, puede suponerse que los pijaos eran descendientes de grupos de cazadores-recolectores que alrededor de 12.000 años a. C. entraron por el istmo de Panamá, se fueron internando por los valles del Cauca y del Magdalena y comenzaron a poblar los espacios que comprenden los actuales departamentos del Tolima y el norte del Huila (Espinosa y Salazar 17)2. De acuerdo con Reichel-Dolmatoff, estos primeros pobladores se ubicaron

I Recientemente algunas publicaciones han demostrado que esta filiación a los karib solo es hipotética, en tanto que no existe una evidencia lingüística suficiente para demostrarlo (Adelaar 53, II 4-II5).

2 Otros autores defienden la idea de que los primeros humanos que llegaron al actual territorio colombiano lo hicieron hace aproximadamente 20.000 años; véase Suaza (23). Con respecto a ello, Juan Rodríguez Freyle afirmaba que los pijaos venían del Darién y se habían asentado en las montañas de la cordillera Central, trabando amistad y parentesco con los paeces. Véase Rodríguez Freyle (347). 
inicialmente en las riberas de los ríos, en especial del Magdalena, por la facilidad de acceso a los recursos que podían obtener de ellos. Sin embargo, en el último milenio a. C. avanzaron hacia las faldas de la cordillera Central y los valles interandinos, se asentaron en ellos y sobrevivieron gracias al desarrollo del cultivo del maíz (Espinosa y Salazar 20; Reichel-Dolmatoff 44-45). Las primeras evidencias humanas en este territorio fueron halladas en el yacimiento El Prodigio, corregimiento de El Limón, municipio del Chaparral, excavadas en 1991. Según las pruebas de carbono I4, estos restos datan de hace 7.370 años. Entre ellos se hallaron artefactos en chert y cuarzo para cazar y para la recolección y triturado de semillas (Espinosa y Salazar i7).

En cuanto a hallazgos arqueológicos pertenecientes propiamente a la cultura pijao, el más significativo de todos fue el descubierto en jurisdicción del actual corregimiento de Rioblanco, al sur del departamento del Tolima, en la vertiente oriental de la cordillera Central. Estos hallazgos, excavados a mediados de la década de 1940, ofrecen una rica información acerca de las formas de poblamiento precolombinas, así como sobre orfebrería, cerámica y herramientas de trabajo que, como se verá más adelante, permiten realizar una serie de análisis sobre los patrones de poblamiento, demografía y relaciones con otros grupos indígenas (Cubillos, "Arqueología" 519-530).

Es importante anotar que los cronistas y los oficiales españoles utilizaron la palabra pijao sin distinción alguna para referirse a cualquiera de los diferentes grupos indígenas que habitaban en aquellas tierras o en las aledańas y que hacían constantes ataques a las ciudades, a las villas y a los pueblos de indios asentados por el régimen español. Sin embargo, como lo han demostrado la arqueología, la etnografía y un estudio más extenso y riguroso de las fuentes manuscritas, en aquella zona habitaron más de 70 grupos indígenas de las familias yanacona, páez, guambiano, quimbaya, panche, pantágora y pijao (Espinosa y Salazar I8-19). Este fenómeno, a saber, el del mito de la gran nación pijao, no es simplemente un error inocente de las crónicas y documentos, sino que se debe a intereses y fenómenos particulares.

Como lo ha indicado Juan David Montoya Guzmán, el criterio de clasificación más utilizado por los españoles fue el de "nación". Bajo este término se agrupaba a los individuos que descendían de un antepasado común, sin importar tanto sus rasgos culturales, su organización política o el territorio que ocupaban. También solía utilizarse el término de "provincia", el cual a diferencia de la actualidad no designaba un territorio físico sino un grupo humano conquistado. Sin embargo, estos términos fueron utilizados por los europeos 
de manera indiscriminada e imprecisa, por lo cual pueden variar mucho en la documentación de la época (Montoya 7I-72).

En el presente caso, a los conquistadores y a sus descendientes no les convenía realizar detalladas descripciones de los grupos nativos de la cordillera, pues ello entorpecía la obtención de los permisos necesarios para llevar a cabo los proyectos de conquista, colonización y pacificación en sus tierras, todo ello en búsqueda de sus intereses particulares. Así, resultaba más fácil etiquetar a todos los indígenas de este territorio como pertenecientes a los "abominables" y "caníbales" pijaos para obrar con mayor libertad, ignorando la legislación que podía proteger a algunos de ellos (Bolaños). Además, en su afán por consolidar el régimen hispánico, los oficiales de la Corona muchas veces aglomeraron de manera forzosa a familias o individuos pertenecientes a diferentes grupos indígenas con el fin de poder administrarlos más fácilmente. Así, terminaron designando a estos grupos con nombres que realmente no les pertenecían ${ }^{3}$.

Por último, debe mencionarse que muchas veces se confundía a los indígenas bajo esta denominación, debido a las constantes alianzas que entre estos grupos se establecían para atacar diferentes regiones. Así, desde los tiempos del adelantado Pascual de Andagoya, pero todavía en las décadas de 1570 y I580, e incluso a comienzos del siglo XVII, coaliciones de indígenas paeces, putimaes, pijaos y toribios atacaban conjuntamente territorios y poblaciones, como las ciudades de San Vicente de Páez, Cartago, San Sebastián de La Plata, Timaná y Neiva, o las provincias de Apirama, Caloto y Guambía ${ }^{4}$.

Uno de los pocos casos en los cuales los oficiales de la Corona se preocuparon por diferenciar a estos grupos indígenas fue el del presidente de la $\mathrm{Au}$ diencia de Santafé, Francisco de Sande. En una carta dirigida al rey, fechada el 2I de octubre de 1597, Sande sostenía que en el territorio de la cordillera Central "ay cinco naciones de indios de guerra los quales salen a hazer daño por toda la

3 Un estudio de este fenómeno en el caso de la provincia de Antioquia en los siglos XVI y XVII puede encontrarse en Montoya y González (96-99). Esta forma de política y administración influyó severamente en las formas de organización prehispánicas, y, por ende, en su economía y demografía. Véase, por ejemplo, la organización de los llamados "archipiélagos verticales" en el imperio incaico y su transformación tras el contacto con los europeos, en John Murra (59-115).

Para los ataques en la época de Andagoya al oriente de Popayán véase Andagoya (I80-18I). Sobre el hostigamiento a las ciudades de San Sebastián de Páez, San Sebastián de La Plata, Timaná y Neiva, y las provincias de Caloto, Apirama y Guambía, véase AGI, P, 233, R.I, ff. 35 r.-42 v. Y las sesiones del cabildo de la ciudad de Popayán desde mayo de 1586 hasta agosto del mismo año, en ACC, $C$, Libros de Belalcázar, ff. 156 r.-193 r. Sobre la presión de putimaes y pijaos a Cartago, véase Ortega Ricaurte (24, II6-II7). 
tierra y por los caminos que van a Popayan para Quito", además de que estos tenían amistades ocultas entre sí para resistir a las jornadas organizadas por las tropas españolas (AGI, $S F$, ramo. I4, n. ${ }^{\circ}$ I40, f. 2 v.).

Sin ignorar las conclusiones que pueden inferirse de las aclaraciones anteriores, varias investigaciones han logrado demostrar que la etnia pijao se dividía en una gran multitud de grupos. Julio César Cubillos alcanza a contar 32 parcialidades (Cubillos, “Apuntes” 53), mientras que Manuel Lucena Salmoral, tras hacer un cruce de información procedente de fuentes documentales, de la crónica de fray Pedro Simón y de las investigaciones de Paul Rivet, enumera un total de 78 pertenecientes a la nación pijao (Lucena I03-IO4). Algunos de los principales grupos eran los cutiva, los ambeima, los amoyá, los otaima, los bulira, los beuni, entre otros.

Sin embargo, y sin dejar de reconocer la gran diversidad étnica dentro de esta misma nación, existían dos grandes subgrupos que pueden diferenciarse, básicamente, por su ubicación geográfica. Estos eran, como los llamaban los españoles, los pijaos de la sierra y los pijaos del llano. Los primeros eran aquellos que habitaban las ásperas sierras de la cordillera Central. Los segundos, conformados por cuatro parcialidades, a saber, coyaimas, natagaimas, guauros y tamagales, eran los que vivían en el valle de Neiva, entre el río Magdalena y las faldas de la cadena montañosa donde residían sus familiares (véase figura I). Ambos conjuntos hablaban la misma lengua y tenían muchas costumbres en común, pero al parecer existía una rivalidad entre ellos desde tiempos prehispánicos. Este fenómeno fue aprovechado por los oficiales españoles para negociar alianzas con los pijaos del llano en busca de su colaboración para enfrentar a sus familiares de las montañas (AGi, $P$ I96, R. 27, f. 989 r.; Borja 478; Simón 444).

Sería contra los pijaos de la sierra, sobre los cuales versa el presente artículo, contra quienes se concentraría la mayoría de los esfuerzos de conquista y pacificación desde mediados del siglo Xvi, pues estos representaban una amenaza al asentamiento, la expansión, la comunicación y el comercio de la sociedad colonial. En cuanto a los pijaos del llano, aunque también asaltaban poblaciones y caminos, fueron dominados intermitentemente hasta su control definitivo bajo el mandato del presidente don Juan de Borja. Así, por ejemplo, a pesar de que los coyaimas habían estado encomendados durante cierto tiempo a hombres como el capitán Alonso Ruiz de Sahajosa, a quien le fueron otorgados por el presidente Antonio González y el gobernador Bernardino Mojica de Guevara, este mismo capitán se vio obligado a volver a reducirlos tiempo 
después a su costa hasta dejarlos en servidumbre y adoctrinamiento (AGN, $H C$ I7, doc. 6, ff. 2IO r.-2II v.).

Otro caso en el que se puede constatar una posición ambivalente por parte de estos indígenas es en las discusiones ocurridas a mediados de 1603 entre los capitanes Pedro Jaramillo de Andrada, de Tocaima, y Gaspar Rodríguez del Olmo, de Ibagué. Según Jaramillo de Andrada, tenía en su poder informaciones en que constaba que los coyaimas "son los que han hecho y hacen los daños que ha habido en esta tierra”. Por su parte, Rodríguez del Olmo, junto con otros vecinos de Ibagué, argumentaba que estos eran indios de paz y que su ayuda era fundamental para el buen desarrollo de la guerra contra los rebeldes de la sierra (Ortega 249-250). Puede que ambos tuvieran razón, pues las identidades y las lealtades de los diferentes sectores que vendrían a formar la sociedad colonial, entre ellos los indígenas, sufrieron variadas reconfiguraciones a lo largo del tiempo. Como acertadamente expresaba el gobernador Domingo de Erazo, estos indios vivían "neutrales, entre la paz y la guerra" (Arango 388).

\section{Habitación}

La zona de habitación de los pijaos de la sierra fueron las montañas de la cordillera Central, tierra sumamente difícil de transitar y explorar por las tropas españolas. Según el presidente de la Real Audiencia de Santafé, don Juan de Borja:

La dicha cordillera donde avitan los Indios Rebeldes es la de mayor aspereza que se conoçe en todas las Indias de altisimos çerros y quebradas hondas, espesissimos bosques y muchos riscos y despeñaderos de muy gran peligro sin apaçibilidad de tierra llana con mas apropiada disposiçion para fieras que para avitaçion de hombres humanos. (AGI, P I96, R. 27, f. 998 v.)

Estas características impidieron a las tropas españolas aprovecharse de dos de sus más valiosos recursos bélicos: los caballos, que tan grandes servicios habían prestado en las conquistas de todas las Indias, y el combate en tierra llana, perfeccionado especialmente en las guerras en Europa. Esto terminó por reconfigurar el tipo de combate de las tropas de la Corona, las cuales debieron adaptarse a una guerra de guerrillas, de desgaste y de supresión de recursos.

Debido al carácter montañoso de su zona de habitación, los pijaos tenían acceso a una variada escala de pisos climáticos que iban desde las calurosas 


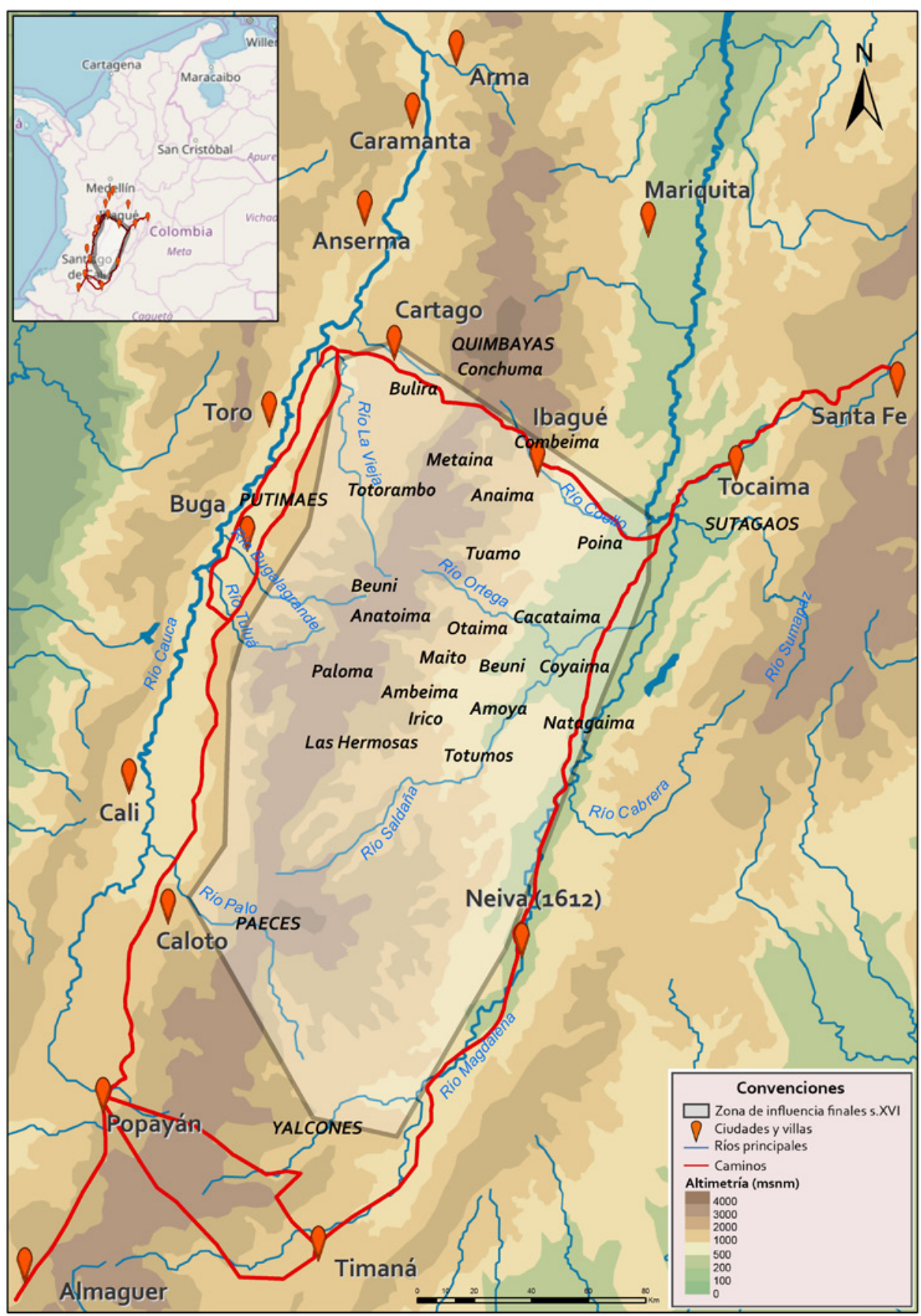

$\leftrightarrow$ FIGURA I.

Territorio de influencia y asentamiento pijao con algunas de sus provincias Fuente: elaboración propia a partir de documentos consultados en AGC, AGI y AGN. 
llanuras cercanas al río Magdalena, pasando por las vertientes de la cordillera Central, hasta llegar a los helados páramos del filo de la cordillera, con alturas de hasta 4.000 m. s. n. m. Además, su tierra contaba con una abundante hidrografía, en la que resaltaban ríos como el Saldaña, el Tetuán o el Barragán, algunos de los cuales transportaban (y continúan haciéndolo) considerables cantidades de oro en sus sedimentos.

En cuanto a la forma y la distribución del tipo de vivienda de los pijaos, parece ser que no solían contar con núcleos poblados, sino que preferían hacer sus casas solitarias y apartadas unas de otras (AGI, $P$ I96, R. 27, f. 988 v.; "Visita de 1560" 39; Ortega I06). Las edificaciones eran construidas en lo alto del monte, para lo cual realizaban aplanamiento de relleno artificial, como el encontrado en las excavaciones de Rioblanco. Según los reportes de Julio César Cubillos, "El espesor de este relleno alcanza una media de I,6o metros, formado por materiales diversos, como arena, arcilla, areniscas tiernas, lascas cuarcíticas, carbón vegetal y gran cantidad de fragmentos de cerámica, esta última localizada hacia la capa vegetal" (Cubillos, "Arqueología” 52I).

Por lo general, sus casas estaban ubicadas con la montaña por espalda y la caída de esta al frente. Solían poner diversas puertas para facilitar el escape de la vivienda en caso de algún ataque. También cavaban cuevas y túneles dentro de las casas, los cuales salían a las barrancas cercanas. Las paredes estaban hechas de barro y madera, o de bahareque blanqueado con arcilla blanca. Los techos eran pajizos, y los había cónicos o de media y dos aguas. Las plantas podían ser rectangulares o cuadradas (Cubillos, "Apuntes" 55; Lucena I09).

Este tipo de poblamiento disperso dificultó a las tropas españolas el sometimiento de la población pijao de manera rápida y efectiva, a diferencia de como lo habían hecho en regiones con grupos humanos que habitaban en centros poblados, como los muiscas. Así, en vez de tener que atacar o tomar un solo foco de poder centralizado, quienes se enfrentaban a este grupo indígena debían recorrer sus accidentadas tierras, buscar sus hogares en la espesura del monte y destruirlos uno por uno. Y aunque en ocasiones anteriores a la derrota definitiva de este grupo indígena a comienzos del siglo XVII se obtuvieron importantes resultados, como lo relata fray Pedro Simón luego de una jorna$\mathrm{da}$ en la cual se quemaron I84 "casas de buenos edificios", lo cierto es que fue casi imposible erradicarlo de manera definitiva, pues los indios, enterados por medio de espías y contactos con otras etnias de las entradas de los españoles, abandonaban sus casas y construían nuevas habitaciones en zonas de mucho más difícil acceso (AGI, $S F, \mathrm{I} 7$, R. I4, n. ${ }^{\circ}$ I4O, f. 2 v.). 
Entender la razón del modelo de dispersión de los indígenas pijaos es complejo. Sus causas pueden ser varias y por el momento parece que solo pueden presentarse algunas hipótesis. La primera de ellas se relaciona con la forma de organización social de este grupo. Como se detallará más adelante, los pijaos no tenían un modelo de sociedad centralizada ni jerarquizada, sino que elegían líderes temporales para el desarrollo de una determinada acción bélica. Por lo demás, durante su cotidianidad, vivían en pequeñas familias aisladas. Así, su dispersión podría deberse a este fenómeno, aunque no puede saberse si como causa o como consecuencia de este.

Por otro lado, podría especularse que esta forma de organización habitacional fue una de las maneras en que este grupo indígena reaccionó para defender su territorio ante el avance del Imperio español, pues parece haber tomado conciencia de la dificultad que esto generaba a las tropas enemigas. A este respecto pueden contrastarse los testimonios de finales del siglo xvi y comienzos del XviI en los que se habla de la dispersión pijao, con lo descrito por fray Pedro de Aguado sobre la expedición del capitán Andrés López de Galarza por aquellas tierras en 1550. Según la crónica de Aguado, el capitán López de Galarza y sus tropas, después de haber pasado por el valle de las Lanzas (lugar donde poco después fundaría la ciudad de Ibagué), se dirigió a la provincia de Metaima, ubicada a tres leguas de allí.

Cuando llegaron, los indios salieron a recibirlos con sus mujeres e hijos, quienes les ofrecieron comida y descanso en sus casas. Según el texto, estas casas, también llamadas "caneyes" por los españoles,

tienen de largo a setenta, ochenta y a cien pasos; son cubiertos de palmicha o de hojas de bihaos, o de paja o de heno que en tierra rasa se cría; en cada bohío de estos vivía casi toda una familia o cognación, porque se hallaba en cada casa de estas haber y morar de cincuenta personas para arriba. (Aguado 625)

Según lo anterior, si bien no puede compararse con la concentración demográfica de cientos y miles de personas, como en el caso muisca, vemos que es algo diferente con respecto a lo que dicen las fuentes posteriores acerca de tan solo unos pocos individuos que habitaban cada casa. Con base en esto, se podría aventurar la idea de que tras el contacto con los europeos y el comienzo de las hostilidades prolongadas (aunque como puede evidenciarse, no siempre fueron encuentros violentos), algunos grupos pijaos optaron por dispersarse y habitar en diferentes partes de la sierra para dificultar los intentos de 
sometimiento. Este tipo de estrategia también fue utilizada en otras latitudes de América, como en el centro y en el sur de Chile por parte de los indígenas mapuche (Boccara 425-46I).

\section{Demografía}

El cálculo de la población de los pijaos de la sierra, tanto durante el último periodo prehispánico como a lo largo del siglo XVI y comienzos del XVII resulta bastante problemático. En primer lugar, porque muchas veces, como ya se demostró, se confunden diferentes etnias indígenas que eran clasificadas indistintamente como pijaos. Y en segundo lugar, debido a que los documentos en los que se realizaban los censos poblaciones por lo general solo se refieren al número de indios tributarios, contando en pocas ocasiones a los ancianos, mujeres y niños, y generando un problema doble. Por un lado, por un conteo parcial de la población. Y por el otro lado, porque al estar los pijaos rebelados (esto es, en guerra y sin pagar tributo), pocas veces se les consideraba en estas pesquisas.

Por último, como ha sido señalado por Langebaek y Tovar Pinzón, surge un inconveniente a la hora de calcular la demografía indígena basándose en el número de tributarios (o en este caso, en indios "de lanza”, es decir, guerreros), pues si se parte de allí va a resultar necesario hacer una multiplicación por las personas dependientes de cada tributario. Así, tradicionalmente se han hecho conteos multiplicando por tres o cuatro personas, pero este proceso está claramente influenciado por el modelo de familia nuclear reciente de Occidente (Langebaek 28). Para obtener cifras más cercanas a la realidad habría que estudiar la estructura familiar de cada región y etnia, y por desgracia, las fuentes no son muy prolíficas este tema en lo concerniente a los pijaos.

Uno de los pocos documentos con los que se cuenta es un censo realizado en r6oz en la encomienda de Ana de Carrión, ubicada en la Mesa de Ibagué, llevado a cabo por el oidor Lorenzo de Terrones. Esta encomienda abarcaba, además de la Mesa, los pueblos de Itaima, Anacaima, Combeima y Buluya. El total de indios de estas parcialidades era de 345, de los cuales 130 eran tributarios, por lo que estos últimos representaban el $37,68 \%$ del total de la población, lo que también puede decirse en términos de que por cada indio tributario había 2,6 no tributarios. Si se hace un conteo más pormenorizado de cada pueblo en 
el que se tuvieron en cuenta los conteos de los integrantes de cada familia, resulta una proporción de entre 2,3 y 2,5 personas por familia (AGN, E 25, doc. 6, ff. II8 r.-I3I v.).

Por otra parte, en 1627 se realizó otra visita por parte del doctor Lesmes de Espinosa Saravia, oidor de la Audiencia de Santafé, a los pueblos de Carima, Chumba y Río de Las Piedras, en jurisdicción de Ibagué, poblaciones que muy posiblemente estaban habitadas por indígenas pijaos pacificados. En la primera de ellas el promedio de integrantes por familia, considerando el total de la población, el número de tributarios y la descripción detallada de cada núcleo familiar hecha por el visitador, era de 2,5 integrantes por familia. En la segunda, corresponde a 3,2. Y en la última, a 3 personas (AGN, VT, I, doc. 2, ff. 248 r.-25I v.; AGN, $V T$, I, doc. 3 , ff. 316 r.-3I8 r.; AGN, $V T$, I, doc. 4, ff. 4I2 r.-4I7 v.).

Si bien se presenta el problema de que se trata de familias muy posiblemente fragmentadas, o cuya organización cambió tras el proceso de conquista y pacificación, es el dato más cercano con el que se cuenta y según el cual se harán las siguientes estimaciones. El mismo visitador realizó diligencias similares el mismo ańo en los pueblos de Anaima, Natagaima, Nataima, Calucaima, Combeima y Tolima. Los promedios de integrantes por familia en cada una de estas poblaciones fueron de 2,2 (en las tres primeras), 2,3, 3, O, 2, O, y I,3, respectivamente (véase tabla I) (AGN, $\mathrm{CO}, 43$, doc. 42 , ff. 788 r.-802 r.).

$\mathrm{Si}$ se considera lo anterior y se tiene presente que estos censos pueden estar alterados por múltiples factores, como los prejuicios de los oficiales a la hora de contabilizar a los indios, los efectos de la guerra contra la población pijao y la correspondiente fragmentación de núcleos familiares, la diferencia entre zonas más o menos afectadas por el conflicto, o la asociación forzada de diferentes parcialidades en pueblos por parte de los visitadores, se obtiene un promedio de 2,4 personas por familia. Para las estimaciones que se realizarán a continuación se utilizará este promedio para procesar los datos poblacionales recopilados correspondientes a los siglos XVI y XVII. Todo esto se hace sin obviar el alto grado de variabilidad y error que puede haber en el cálculo del número de integrantes de un grupo indígena, difícil de rastrear con precisión en las fuentes.

Muchas y variadas son las opiniones sobre el tamaño de la población pijao al momento de la conquista. En i688 Lucas Fernández de Piedrahita calculaba su número total en $\mathbf{2 0}$.000, mientras que fray Alonso de Zamora aseguraba que para 1565 había I8.000 indios de guerra, lo cual en el segundo caso resultaría en un total aproximado de 43.200 habitantes si se aplica la multiplicación de 
$\rightarrow$ TABLA I.

Promedio de integrantes por familia en parcialidades pijao

\begin{tabular}{|c|c|c|c|}
\hline $\begin{array}{l}\text { Población o } \\
\text { parcialidad }\end{array}$ & Encomendero & $\begin{array}{l}\text { Promedio } \\
\text { integrantes } \\
\text { por familia }\end{array}$ & $\begin{array}{c}\text { Año } \\
\text { información }\end{array}$ \\
\hline Mesa de Ibagué & Doña Ana de Carrión & 2,5 & 1603 \\
\hline Itaima & Doña Ana de Carrión & 2,5 & 1603 \\
\hline Buluya & Doña Ana de Carrión & 2,3 & 1603 \\
\hline $\begin{array}{l}\text { Anacaima y } \\
\text { Combeima }\end{array}$ & Doña Ana de Carrión & 2,5 & 1603 \\
\hline Carima & Sebastián de Porras Salazar & 2,5 & 1627 \\
\hline Chumba & Francisco Vicario & 3,2 & 1627 \\
\hline Río de las Piedras & Isabel Enríquez de Novia & 3,0 & 1627 \\
\hline $\begin{array}{l}\text { Anaima y } \\
\text { Natagaima }\end{array}$ & Blas Cobo & 2,2 & 1627 \\
\hline Nataima & Don Bartolomé de Meneses & 2,2 & 1627 \\
\hline Icataima & Manuel de Lorenzana & 2,3 & 1627 \\
\hline Calucaima & Doña María de Guzmán & 3,0 & I627 \\
\hline Combeima & Francisco de Leuro & 2,0 & 1627 \\
\hline Tolima & Diego del Pulgar & $\mathrm{I}, 3$ & 1627 \\
\hline
\end{tabular}

Fuente: elaboración propia con base en AGN, E, 25, doc. 6, ff. II8 r.-I3I V.; AGN, VT, I, doc. 2, ff. 248 r.-25I V.; AGN, VT, I, doc. 3, ff. 316 r.-3I8 r.; AGN, VT, I, doc. 4, ff. 4I2 r.-4I7 V.; AGN, CO, 43, doc. 42 , ff. 788 r. -802 r.

2,4 personas dependientes por cada indígena guerrero (Lucena IO2) 5 . En I560, luego de una visita anónima, se estimaba que los indígenas rebelados de los términos de las ciudades Almaguer, Popayán, Timaná, Neiva, Cartago, Mariquita y Tocaima sumarían alrededor de 44.000 personas ("Visita de 1560 " 32-72).

5 Cabe mencionar que Lucena Salmoral utiliza la tradicional multiplicación por aproximadamente cuatro personas, por lo que concluye que Zamora hacía referencia en este caso a un número de entre unos 50.000 y 70.000 habitantes. 
Para 1597 el presidente Francisco de Sande estimaba el número de estos entre 5.000 y 6.000 individuos (AGI, $S F$, I7, R. I4, n. ${ }^{\circ}$ I 40, f. 2 v.).

Sin embargo, y compartiendo la opinión del historiador Manuel Lucena Salmoral, algunas de estas cifras (exceptuando tal vez la del presidente Sande) se presentan como exageradas si se tienen en cuenta las informaciones de primera mano aportadas por soldados que participaron directa y activamente en la guerra contra estos indígenas. En I603, el capitán Diego de Bocanegra manifestaba que en toda la tierra no habría más de 1.50o indios de guerra (Ortega Io6). En su informe de 1606, el gobernador Domingo de Erazo afirmaba que "según la más cierta relación y tanteo serán todos seys mil yndios de guerra” (Arango 388). Para 1608, el presidente de la Audiencia, don Juan de Borja, comunicaba al rey que "segun la mas comun opinnion y lo que los prisioneros deponen serian entonçes dos mill yndios gandules" (AGI, $P$, I96, R. 27, f. 998 v.).

Finalmente, en I613, según las informaciones de varios capitanes veteranos, recopiladas por el oidor de la Audiencia de Santafé, Juan de Villabona, se calculaba que entre todos los indios que habían sido asesinados y capturados sumarían alrededor de 2.000 o 3.000 personas, aparte de los que habrían muerto por las enfermedades y el hambre (AGI, $P$, I96, R. 28, ff. I036 v., I038 v., IO4I r.-IO4I v.). Considerando que estas últimas cifras se refieren únicamente al número de indígenas guerreros, y multiplicando estas cantidades de acuerdo con la idea de que había 2,4 personas por cada indio gandul, o de lanza, sumarían aproximadamente 6.000 , tal vez un poco más, de indígenas pijaos que vivían a comienzos del siglo Xvir. Es probable que esta cifra fuera algo mayor en tiempos prehispánicos.

Además de esta información, existen otros datos que pueden ayudar para hacerse una mejor idea de la densidad demográfica de los pijaos. Parece ser que incluso desde tiempos prehispánicos esta región no tuvo una muy alta densidad poblacional. Según Hermes Tovar Pinzón, quien calcula la población indígena del actual territorio colombiano para el año de I5OO en II.332.823 habitantes, solo el 6,I4\% de estos vivían en los Andes centrales, esto es, alrededor de 320.605 personas pertenecientes a diferentes etnias de esta región (Tovar III-II2) ${ }^{6}$.

6 Algunas investigaciones discrepan sobre los cálculos presentados por Tovar Pinzón, en cuanto que no considera algunos datos de regiones como la Amazonia, la cual contaba, según algunos estudios, con varios millones de pobladores en el siglo XVI. Véase, por ejemplo, Ribeiro (79), quien calculaba la población en cinco millones. 
$\rightarrow$ TABLA 2 .

Población pijao

\section{Informante}

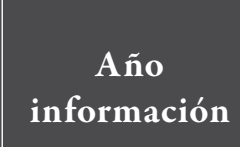

Cantidad de tributarios o

guerreros
Cantidad total

(multiplicando

por 2,4 )

Antes del contacto con los españoles

\begin{tabular}{|l|c|c|c|}
\hline $\begin{array}{l}\text { Lucas Fernández de } \\
\text { Piedrahita }\end{array}$ & I688 & I20.000 (total) & I20.000 \\
\hline \multicolumn{2}{|l|}{ Después del contacto con los españoles } \\
\hline Relación anónima & I560 & 44.000 (total) & 44.000 \\
\hline Fray Alonso de Zamora & 1565 & 18.000 & 43.200 \\
\hline Francisco de Sande & 1597 & $5.000-6.000$ & I3.200 aprox. \\
\hline Diego de Bocanegra & 1603 & 1.500 & 3.600 \\
\hline Domingo de Erazo & 1606 & $6.000($ total) & $6.000^{*}$ \\
\hline Juan de Borja & 1608 & 2.000 & 4.800 \\
\hline Juan de Villabona & 1613 & $2.000-3.000$ & 6.000 aprox. \\
\hline
\end{tabular}

*Aunque el gobernador Erazo aclara que para este conteo se trata de 6.000 "yndios gandules", si se compara con las estimaciones más cercanas parece más bien que se refería al total de indios pijaos.

Fuente: elaboración propia a partir de AGN, P, I96, r. 27 y r. 28.

Por otras fuentes puede concluirse también que, de esta masa poblacional, la etnia de los pijaos de la sierra representaba un pequeño número. Según reporta Juan de Castellanos al relatar los enfrentamientos entre yalcones y españoles a finales de la década de 1530 y comienzos de la de 1540 , cerca de la ciudad de Timaná, cuando la célebre cacica Gaitana buscó la ayuda de los pijaos para su venganza, estos se encontraban ya en aquel entonces "cuasi consumidos" debido a, según este cronista, guerras civiles y haberse comido unos a otros. En dicha ocasión acudieron a ayudar con 3.000 guerreros (Castellanos 915-916). En otro documento, en este caso una información mandada a realizar en la ciudad de San Sebastián de La Plata por el gobernador de Popayán don Sancho García del Espinar, en noviembre de 1577 , varios testigos tratan acerca del poco número de los pijaos, incluso en sus propias provincias. 
Según Marcos Ortiz, vecino y regidor de la ciudad, y quien afirmó haber recorrido varias veces las tierras de los pijaos, no había allí casi ninguno de estos indios, sino que los que andaban por aquellos lugares eran casi todos indios paeces, emparentados con los pijaos. Dijo además que, según su propia experiencia, antes de que los pijaos y los paeces estuvieran aliados, los primeros solo hacían asaltos con máximo 35 guerreros y casi siempre terminaban huyendo, lo que parece una nimiedad en comparación con las grandes juntas que se hacían en la provincia de Toboima de más de 2.000 indígenas, en su mayoría paeces (AGI, $P$ 233, R. I, ff. 80 r., 86 v.). Estas afirmaciones fueron respaldadas por los testimonios del capitán Fernando Díaz de la Fuente y de Juan Calderón, este último vecino y regidor de la ciudad, quien aseguraba que

los dichos yndios pixaos son pocos y que solos ellos sin otro fabor no fueran parte para hazer tan grandes daños como an echo y hazen por el fabor y ayuda que tienen de los yndios caziques y principales de las dichas probincias de paez como segun se contiene por cosa muy cierta y aberiguada de las probincias de toboyma donde se haze la junta de los yndios paezes para dar fabor y ayuda a los dichos pixaos sienpre que quieren salir a hazer sus saltos por quanto los dichos paez es jente muy belicosa y valiente para la guerra. (AGI, $P, 233$, R. I, f. 98 v.)

¿A qué podría deberse esta pronunciada disminución de la población pijao? ¿Era en verdad producida, como decía Castellanos, por guerras civiles y una antropofagia desmesurada? Lo cierto es que no solo la población pijao, sino en general toda la de los Andes centrales tuvo una vertiginosa caída durante el siglo XVI y comienzos del Xvir. Según algunas investigaciones, las tasas de crecimiento de la población indígena que habitaba en las jurisdicciones de las ciudades donde los pijaos tenían presencia o influencia fueron siempre negativas durante el siglo XVI y XVII, y sus valores variaron desde el $-3,0 \%$ hasta un -II, $4 \%$ (Tovar I35).

Como ya se mencionó, gran parte de este descenso pudo deberse a la modificación de las formas de vida tradicionales a raíz de la reorganización espacial llevada a cabo por los espańoles. Era muy usual, por ejemplo, que muchos indígenas murieran cuando se los trasladaba a un lugar con un clima totalmente diferente al de su origen. Ya en 1549 Sebastián de Magaña, tesorero de Popayán, mencionaba que en el camino que bajaba de Timaná a Tocaima, atravesando el valle de Neiva, muchos indios morían o enfermaban de gravedad por no estar acostumbrados a las temperaturas (Colmenares 30-3I). Además, el 
haber desmembrado la estructura familiar indígena también fue un factor que influyó fuertemente en las tasas de natalidad.

Otro aspecto por considerar es el de las epidemias traídas por los europeos, que en muchas ocasiones llegaban antes que los propios conquistadores, y a las cuales los cuerpos de los indígenas eran especialmente susceptibles. Según la visita anónima de 1560, durante el año de 1559 hubo una terrible epidemia en toda la jurisdicción del Nuevo Reino, la cual afectó severamente a las tierras cercanas a Ibagué (esto es, tierras de pijaos), provocando la muerte de muchas personas a causa de viruelas y sarampión ("Visita de 1560 " 63). Según los vecinos de la ciudad, cuando esta se pobló en 1550 había alrededor de 10.000 indígenas, pero tan solo diez años después solo quedaban cerca de 2.700 ("Visita de 1560" 6I-62, 64).

Con respecto a las hipótesis de una disminución poblacional a causa de un continuo estado de guerra y un voraz canibalismo, estas deben analizarse a la luz de diferentes consideraciones aportadas por varios antropólogos e historiadores. Según algunas tendencias, los constantes conflictos bélicos entre las comunidades prehispánicas se debieron principalmente a la captura de prisioneros para comerlos o realizar trabajos forzados, a la expansión de los nacientes cacicazgos, y a una competencia por la disponibilidad de recursos en la región. Con respecto al canibalismo, se cree que se practicaba con frecuencia para fines ceremoniales y rituales, o como una forma de suplir la falta de proteínas y alimentos (Jaramillo 44, 55, 60-6I, 65).

Sin embargo, al contrastar estas teorías con un análisis más profundo de la documentación y de los restos arqueológicos, se presentan varias inconsistencias. En primer lugar, la tierra en la que habitaban los pijaos era bastante rica en recursos, especialmente porque podían acceder a una variada cantidad de climas y altitudes que podían proveerles los alimentos necesarios. Es más, al parecer sus cultivos eran muchos y diversos, según se puede concluir de las extendidas campañas de talas y quemas que los soldados españoles hicieron de ellos en sus expediciones.

Además, también se tiene evidencia de que tuvieron contactos comerciales y culturales con diferentes grupos como los quimbayas, los muiscas o las culturas de San Agustín, lo que podría haberles proveído de algunos de los elementos que no conseguían en su tierra ("Visita de 1560" 45; Cubillos, "Apuntes" 62-63; Cubillos, "Arqueología" 523, 528). Por su parte, la idea de la competencia por el avance y la formación de los cacicazgos parece no tener mucha cabida en el caso pijao, pues como se señaló, este grupo indígena era bastante fragmentario y no tenían una organización jerárquica. 
Con respecto al canibalismo, tan repetido en las fuentes españolas, puede evidenciarse que este era practicado especialmente con etnias diferentes de la propia. Así, los pijaos solían atacar principalmente a los duhos y a los badahujos, ubicados en la margen oriental del río Magdalena, a la altura del valle de Neiva, y a los sutagaos, quienes residían al sur de la tierra de los muiscas, a la altura del río Cuja (Cubillos, "Apuntes" $5 \mathrm{I}, 53$ )7. Sin embargo, sus principales víctimas eran los mismos españoles o los indios de servicio que trabajaban para ellos en sus estancias y encomiendas. De esta forma, la idea de un descenso demográfico con base en un canibalismo interno entre los pijaos no parece tener mucha validez, aunque esto no pretende negar la existencia de la antropofagia en este grupo.

De lo anterior puede concluirse que, en primer lugar, los pijaos de la sierra no eran un grupo muy numeroso, y, en segundo lugar, que la baja de su población se debió principalmente a factores como las enfermedades o la modificación de sus tradiciones organizacionales y familiares.

\section{Organización social y actividades de subsistencia}

La estructura social de los pijaos es especialmente difícil de comprender debido a las concepciones que durante aquellos tiempos reinaban en la mente de quienes constataron por escrito alguna mención de su tipo de organización. De manera casi unánime, y siguiendo una tradición que se remontaba hasta tiempos de la Grecia clásica, los europeos que llegaron a América, incluyendo a quienes lucharon contra los pijaos a comienzos del siglo XVII, tenían una concepción muy poco flexible acerca de qué podía considerarse verdaderamente una sociedad, es decir, qué separaba a una comunidad en estado de naturaleza puro de una más "civilizada". Su visión era que todo grupo humano que fuese considerado "sociedad" debía encontrarse organizado bajo la división de señores y súbditos. De esa forma, se concluía que si un grupo humano carecía de esta característica, no contaba con un gobierno o un Estado, por ende, no podía ser una sociedad y pasaba a ser considerado un aglomerado de salvajes "sin fe, sin ley, y sin rey" (Clastres I84-186).

$7 \quad$ Uno de los pocos casos de canibalismo entre grupos pijaos es el de dos indígenas coyaimas de la compañía del capitán Juan Bautista de los Reyes, quienes durante la campaña de 1609 o I6ro tomaron y comieron a un bebé de los pijaos de la sierra. Véase Simón (437). 
Por lo anterior, a diferencia de las detalladas descripciones acerca de la vida y la sociedad de pueblos como los mexicas o los habitantes del Tahuantinsuyo, en el caso de los pijaos se cuenta con textos mucho más escuetos y menos detallados, debido a los prejuicios europeos. Así, más que pesquisas sobre los grupos humanos, se hacían sobre todo de sus recursos naturales, en especial de la existencia de yacimientos de metales preciosos. Sin embargo, y teniendo presente esta problemática, es indudable que las fuentes permiten una cierta aproximación a la organización social de los pijaos.

A diferencia de otras sociedades indígenas, la estructura de la comunidad pijao no era piramidal o jerárquica. Tal vez la única excepción era una especie de respeto que tenían a los mohanes, aunque este tampoco era demasiado estricto, pues en caso de que se equivocaran en sus predicciones, el pueblo podía golpearlos e incluso matarlos (AGI, $P$, I96, R. 27, f. 990 r.). Según una carta del I2 de octubre de 1607 del Cabildo de Ibagué al rey, "esta gente nunca se gobierna por caciques ni señores sino por behetría sin tener a quien respetar ni obedecer ni de quien con seguridad nos podamos prometer seguridad en sus contratos" (AGI, $S F, 65$, n. ${ }^{\circ}$ 56, f. 2 r.). Similares opiniones ya habían sido emitidas ańos atrás, como en la visita anónima de 1560 , en las que era normal que se dijera de los indios de estas regiones que "No ay caciques ni señores naturales, es todo behetría y gente mal vestida". También el presidente don Juan de Borja repetiría esta idea en sus diferentes cartas e informes al rey (AGI, $P$, I96, R. 27, f. 99I v.; AGI, $S F$, I8, R. 8, n. 68 , f. I v.).

Lo anterior dificultaba en extremo su conquista y sumisión por parte de las tropas españolas, que no podían eliminar o aliarse con un líder mayor para facilitar y acelerar la victoria, tal como habían hecho con Moctezuma en Nueva España y con Atahualpa en el Perú. Aun así, había ocasiones en que las diferentes parcialidades indígenas se reunían en una junta y elegían a una especie de líder o capitán temporal para que dirigiera una determinada acción bélica que tuvieran planeado llevar a cabo. Lo usual era que se escogiera entre los hombres más valientes que hubiese, con la claridad de que una vez terminada la campaña este debía retirarse de su cargo (Lucena II3-II5). Ello no significaba que el hombre designado no pudiera ser relegido para futuras acciones, como sucedió en el caso del afamado "cacique" Calarcá, que comandó diversos ataques, entre los que se encuentran el asalto a Ibagué a mediados de 1606 y el asedio al fuerte del capitán Diego de Ospina en 1607 (Simón 376, 409-4I4).

Con respecto a las mujeres, parece que su papel era más bien de madres y encargadas de las tareas del hogar, bastante celadas por sus esposos. Solían 
casarse poco después de haber menstruado por primera vez, y tras pasar la primera noche con sus maridos se soltaban unas ligaduras que llevaban en los tobillos y muñecas para indicar su nuevo estatus (AGI, $P$, 196, R. 27, f. 990 v.). Sin embargo, y si se considera que los pijaos participaron y apoyaron la rebelión de los yalcones, dirigida por la cacica Gaitana, quien fue personalmente a buscar su ayuda, puede pensarse que, o bien su actitud hacia las mujeres era diferente a la consignada por los españoles, o que podía variar con respecto a otros grupos.

En cuanto a la principal fuente de alimentos de los pijaos, puede decirse que provenía de la agricultura, la cual era practicada en lugares apartados y ocultos para asegurar sus cosechas de manos enemigas. Para sus labranzas utilizaban raíces y palos a modo de azadón (Cubillos, "Apuntes" 60). El maíz preponderaba sobre el resto de sus cultivos, pues lo sembraban durante todo el año. Este era consumido principalmente cocido o tostado, aunque también hacían harina para fabricar bollos. De él también extraían lo necesario para la elaboración de masato y chicha, esta última considerada "su prinçipal sustento y regalo" y "sin que en vida y muerte aya de aver cosa que no sea autoriçada con la chicha" (AGI, $P$, 196, R. 27, ff. 99I r.-99I v.). También sembraban y consumían otros alimentos como frijol, arracacha, yuca, papa, aguacate, papayuela, ciruela y piña (aGi, $P$, I96, R. 27, f. 99I v.; Simón 43I). En cuanto a carne o pescado, no obtenían mayor cosa de la caza, aparte de algún venado o pájaro que mataban con cerbatanas.

Otra de las actividades a las cuales se dedicaban los indígenas era la minería, mayoritariamente de aluvión, aunque al parecer los pijaos del norte practicaban también la de veta, con lo cual hacían joyas que deleitaron a los conquistadores españoles (Cubillos, "Apuntes" 6I). Estas piezas de oro eran especialmente deseadas, pues se caracterizaban por el empleo de oro de alta ley, es decir, sin muchas aleaciones con otros metales, a diferencia de como se hacía en otras partes del actual territorio colombiano (Plazas y Falchetti i6). Descendiendo por el costado oriental de la cordillera Central corrían algunos ríos como el Saldaña, el Coello y el Irco, que hasta hoy día siguen arrastrando importantes cantidades de oro en sus arenas, los cuales representaban la mayor fuente de este mineral desde tiempos prehispánicos.

Los artículos de oro fueron utilizados por los pijaos para confeccionar adornos corporales como narigueras, pectorales, pendientes o cuentas de collar (Cubillos, "Apuntes" 6I). También lo usaron como artículo de intercambio con otros grupos indígenas. La llamada "feria de Coyaima", situada a orillas del río Saldaña, o el mercado ubicado en el actual municipio de Aipe, en el 
departamento del Huila, eran importantes centros de comercio entre los pijaos y otros grupos vecinos que, por lo general, se comunicaban gracias al río Magdalena. En dichos eventos se realizaban todo tipo de intercambios. Los muiscas, por ejemplo, asistían allí para cambiar sus mantas, sal y esmeraldas por las joyas de oro pijao (Cubillos, "Apuntes" 62-63). El oro también fue implementado para comprar la paz de los españoles, como ocurrió en el caso del cacique Matora, quien durante el paso de un grupo de soldados por la provincia de Amoyá en 1556, comandados por Francisco de los Barrios, sobrino del primer arzobispo del Nuevo Reino y subordinado del capitán Francisco de Trejo, les ofreció un presente "que era hasta de mil pesos en chagualas y joyas de su usanza" para evitar un enfrentamiento (Simón 334).

\section{Conclusiones}

A pesar de que el pueblo pijao ha sido uno de los más renombrados en la historia del país, realmente es poco lo que se conoce sobre ellos, a excepción de la guerra que sostuvieron contra el régimen español entre los siglos XVI y XVII. Por tal motivo, este artículo ha ahondado en cuestiones como su origen, su ubicación geográfica, sus formas de habitación y poblamiento, su demografía, su organización social y sus actividades de subsistencia, para poder llegar a una mejor comprensión de su sociedad.

Estos elementos permiten considerar mejor el actuar de los pijaos en los procesos históricos en que tomaron parte, como su famosa guerra. Así, por ejemplo, por medio de estudios demográficos rigurosos puede entenderse mejor la repercusión del conflicto y la desestructuración de este grupo a raíz de aquel. Además, el artículo abre otras posibilidades de estudio que permitan entender mejor el mundo colonial en los siglos XVI y XVII. Una de estas es la investigación para diferenciar mejor los diferentes grupos indígenas del territorio, con el fin de dejar de replicar muchos de los prejuicios de clasificación presentes en las fuentes españolas y tener una perspectiva más acertada del actuar de diferentes grupos indígenas. 


\section{. \\ B I B L I O G R A F í A}

\section{F U E N T ES PR I MAR IA S}

\section{A. Archivos}

Archivo General de Indias, Sevilla, España (AGI)

Patronato (P), 196 y 233.

Santa Fe (SF), I7, I8 y 65 .

Archivo General de la Nación, Bogotá, Colombia (AGN)

Curas y Obispos (CO), 43.

Encomiendas (E), 25.

Historia Civil ( $\mathrm{HC}), \mathbf{1 7}$.

Visitas Tolima $(V T)$, I.

Archivo Central del Cauca, Popayán, Colombia (ACC)

Cabildo (C)

Libros de Belalcázar.

\section{I F U E N T ESS E C U N D A R I A S}

Adelaar, Willem F. H. The Languages of the Andes. Nueva York: Cambridge University Press, 2004.

Aguado, Fray Pedro de. Historia de Santa Marta y Nuevo Reino de Granada, tomo I. Madrid: Establecimiento Tipográfico de Jaime Ratés, I916.

Andagoya, Pascual de. "Relacion que da el adelantado de Andaboya de las tierras y probincias que abaxo se ara mencion”. Relaciones y visitas a los Andes, tomo I, por Hermes Tovar Pinzón. Bogotá: Universidad de Los Andes, Colombia, 2010, pp. 103-186.

Arango Puerta, Mauricio, transcriptor. "Informe de Domingo de Erazo sobre la guerra contra los indios pijaos, 1606 ”. Historia y Sociedad, n. ${ }^{\circ} 33,2017$, pp. 380-396. https:// doi.org/I0.15446/hys.n33.63306

Boccara, Guillaume. "Etnogénesis mapuche: resistencia y restructuración entre los indígenas del centro-sur de Chile (siglos XVI-XvIII)". The Hispanic American Historical Review, vol. 79, n. ${ }^{3}$, 1999, pp. 425-461. https://doi.org/10.1215/00182168-79.3.425 
Bolaños, Álvaro Félix. Barbarie y canibalismo en la retórica colonial: los indios pijaos de fray Pedro Simón. Bogotá: Cerec, 1994.

Borja, Juan de. "Don Juan de Borja informa sobre la guerra contra los indios Pijao [25 de mayo de r6ro]". Relaciones y visitas a los Andes, tomo IV, por Hermes Tovar Pinzón. Bogotá: Universidad de Los Andes, Colombia, 20ı, pp. 473-484.

Castellanos, Juan de. Elegías de varones ilustres de Indias. Bogotá: Gerardo Rivas Moreno, 1997.

Clastres, Pierre. Investigaciones en antropología politica. Barcelona: Gedisa, I98I.

Colmenares, Germán. Historia económica y social de Colombia, I537-I719. Medellín: La Carreta, 1978 .

Cubillos, Julio César. "Apuntes para el estudio de la cultura pijao”. Boletín de Arqueología, vol. 2, n. ${ }^{\circ}$ I, 1946, pp. 47-8I.

---. “Arqueología de Rioblanco (Chaparral, Tol.)”. Boletín de Arqueología, vol. I, n. 6 , i945, pp. 519-530.

Espinosa Rico, Miguel Antonio y Salomón Salazar Morales. Poblamiento y espacios en el Alto Magdalena-Tolima. La configuración histórica del territorio. Ibagué: Centro de Estudios Regionales de la Universidad del Tolima, 2003.

Friede, Juan. Los quimbayas bajo la dominación española. Bogotá: Carlos Valencia Editores, 1982.

Jaramillo, Luis Gonzalo. "Guerra y canibalismo en el valle del río Cauca en la época de la conquista española”. Revista Colombiana de Antropología, vol. XXXII, 1995, pp. 4I-84.

Langebaek, Carl Henrik. "Reconstrucciones demográficas de la población indígena de Colombia antes y después de la Conquista española”. La economía colonial de la Nueva Granada, editado por Adolfo Meisel Roca y María Teresa Ramírez G. Bogotá: Fondo de Cultura Económica, Banco de la República, 20I5, pp. 2I-6I.

Lucena Salmoral, Manuel. Presidentes de Capa y Espada (I605-1628). Historia Extensa de Colombia, vol. III, tomo 2, coordinado por Luis Martínez Delgado. Bogotá: Ediciones Lerner, 1965.

Montoya Guzmán, Juan David. “Las más remotas tierras del mundo: historia de la frontera del Pacífico, I573-1687”. Tesis doctoral en Historia de América Latina, Universidad Pablo de Olavide, 2014.

Montoya Guzmán, Juan David y José Manuel González Jaramillo. Indios, poblamiento y trabajo en la provincia de Antioquia, siglos XVI y XVII. Medellín: Universidad Nacional de Colombia, Sede Medellín, Facultad de Ciencias Humanas y Económicas, 20 o. 
Murra, John. Formaciones económicas y politicas del mundo andino. Lima: Instituto de Estudios Peruanos, 1975.

Ortega Ricaurte, Enrique. Los inconquistables. La guerra de los pijaos. Bogotá: Archivo Nacional de Colombia, I949.

Plazas de Nieto, Clemencia y Ana María Falchetti de Sáenz. "Orfebrería prehispánica de Colombia”. Boletín Museo del Oro, n. ${ }^{\circ}$, I978, pp. I-53.

Reichel-Dolmatoff, Gerardo. “Colombia indígena, período prehispánico”. Nueva Historia de Colombia, vol. I, dirigida por Jaime Jaramillo Uribe. Bogotá: Planeta, 1989, pp. 27-68.

Restrepo Canal, Carlos. "Gobierno de don Juan de Borja en el Nuevo Reino de Granada, 1605-1628”. Revista de Indias, n. 50, 1952, pp. 729-744.

Ribeiro, Berta G. Amazônia urgente: cinco séculos de história e ecologia. Belo Horizonte: Itatiaia, 1992.

Rodríguez Freyle, Juan. El carnero. Caracas: Biblioteca de Ayacucho, 1979.

Simón, Fray Pedro. Noticias historiales de las conquistas de Tierra Firme en las Indias Occidentales, tomo vi. Bogotá: Biblioteca Banco Popular, i98I.

Suaza Español, María Angélica. "El territorio de Neiva: desde los cazadores-recolectores hasta los aguerridos pijaos". Historia comprehensiva de Neiva, tomo I, editado por Bernardo Tovar Zambrano y Reynel Salas Vargas. Neiva: Alcaldía de Neiva, Academia Huilense de Historia, 2012, pp. 23-67.

Tovar Pinzón, Hermes. "Las cifras y los métodos en la reconstrucción de la población colombiana”. La economía colonial de la Nueva Granada, editado por Adolfo Meisel Roca y María Teresa Ramírez G. Bogotá: Fondo de Cultura Económica, Banco de la República, 2015, pp. 104-143.

Velásquez Arango, Juan José. “La guerra contra los indígenas pijaos: financiamiento, organización militar y vida cotidiana, I550-1615”. Tesis de Maestría en Historia, Universidad Nacional de Colombia, Sede Medellín, 2018.

"Visita de 1560". No hay caciques ni señores, transcripción e introducción de Hermes Tovar Pinzón. Barcelona: Sendai, I988, pp. 21-120. 